\title{
Mythos
}

Rivista di Storia delle Religioni

$10 \mid 2016$

Varia

\section{Franz Cumont, Recherches sur le symbolisme funéraire des Romains}

Volume édité par Janine et Jean-Charles Balty, avec la collaboration de Charles Bossu, Nino Aragno Editore (Bibliotheca Cumontiana, Scripta Maiora IV), Academia Belgica, Institut historique belge de Rome, 2016, pp. CLXV-548, ISBN 978-90-74461-78-8, € 90

\section{Francesco Massa}

\section{(2) OpenEdition \\ Journals}

\section{Edizione digitale}

URL: http://journals.openedition.org/mythos/419

DOI: $10.4000 /$ mythos. 419

ISSN: 2037-7746

\section{Editore}

Salvatore Sciascia Editore

\section{Edizione cartacea}

Data di pubblicazione: 1 dicembre 2016

Paginazione: 231-232

ISBN: 978-88-8241-476-4

ISSN: $1972-2516$

\section{Notizia bibliografica digitale}

Francesco Massa, «Franz Cumont, Recherches sur le symbolisme funéraire des Romains », Mythos [Online], 10 | 2016, online dal 24 septembre 2019, consultato il 24 septembre 2020. URL : http:// journals.openedition.org/mythos/419; DOI : https://doi.org/10.4000/mythos.419 


\title{
Janine \& Jean-Charles Balty (éds)
}

\author{
Franz Cumont, Recherches sur le symbolisme funéraire des Romains \\ Volume édité avec la collaboration de Charles Bossu, Nino Aragno Editore (Bibliotheca Cumontiana, Scripta Maiora IV), \\ Academia Belgica, Institut historique belge de Rome, 2016, pp. CLXV-548, ISBN 978-90-74461-78-8, € 90
}

Francesco Massa - Université de Genève - Francesco.Massa@unige.ch

La morte ha prodotto ogni sorta di racconti sugli dèi Mani [...]. A volte si ammette l'immortalità dell'anima (inmortalitatem animae), a volte la metempsicosi (transfigurationem), a volte si attribuisce ai morti la facoltà di percepire e si onorano i Mani e si fa un dio di colui che non è neanche più un uomo. Così nel libro della Naturalis historia dedicato all'antropologia, Plinio il Vecchio descrive la pluralità di credenze che i Romani del I secolo d.C. avevano sviluppato sul tema della vita oltre la morte (VII, 188). Se si volesse riassumere in una sola frase, si potrebbe dire che la ricerca di Franz Cumont si propone di studiare questa varietà di credenze attraverso l'analisi delle immagini in contesto funerario.

F. Cumont concluse le sue Recherches sur le symbolisme funéraire des Romains nell'agosto del 1941 e il libro fu pubblicato nel 1942, a Parigi, in una fase tragica della storia europea e in una Francia umiliata e occupata dalle truppe del Reich. Com'è detto chiaramente nella prefazione di Cumont, il volume si propone uno studio del materiale archeologico (soprattutto le raffigurazioni delle stele e dei sarcofagi) al fine di comprendere il rapporto tra i miti rappresentati nei monumenti funerari e le credenze nella vita dopo la morte. Le opere studiate provengono, per lo più, dalle botteghe artistiche di Roma, attribuendo di fatto una certa omogeneità al materiale raccolto nelle pagine del Symbolisme. I cinque capitoli che compongono l'opera analizzano la concezione filosofica dei due emisferi della terra simbolizzati dalla figura dei Dioscuri (capitolo 1), l'atmosfera intesa come il luogo in cui abitano le anime (capitolo 2), la Luna come sede dei morti (capitolo 3), il rapporto tra il culto delle Muse e l'idea dell'immortalità (capitolo 4), il riposo dei morti nel mondo celeste (capitolo 5). Anche solo il semplice elenco dei temi studiati consente di sottolineare l'importanza fondamentale giocata dalla lettura allegorica nel metodo cumontiano di interpretazione delle immagini: le speculazioni pitagoriche e stoiche sono alla base della riflessione sulle immagini, come espresso chiaramente nell'introduzione dell'autore.

Il volume curato da Janine e Jean-Charles Balty (con la collaborazione di Charles Bossu) si inserisce nell'importante progetto di riedizione delle opere di Franz Cumont iniziata nel 2006, e fa seguito alla pubblicazione di Les religions orientales dans le paganisme romain (a cura di C. Bonnet e F. van Haeperen), Lux Perpetua (a cura di B. Rochette e A. Motte) e Les mystères de Mithra (a cura di N. Belayche e A. Mastrocinque). Seguendo lo schema utilizzato anche per le altre opere della collezione promossa dall'Academia Belgica di Roma, la riedizione è preceduta da una ricca e ampia introduzione storiografica che occupa le prime 150 pagine del volume, su cui concentreremo la nostra attenzione per questa recensione. Agli indici già presenti nell'edizione del 1942 (indice generale e indice topografico), la riedizione ha aggiunto un utile indice delle fonti antiche, realizzato da Matthieu Soler.

Sotto il titolo Genèse, réception et avenir des Recherches sur le symbolisme funéraire des Romains, i curatori propongono al lettore non soltanto un percorso nella struttura e nei meandri dell'opera cumontiana, ma anche una preziosa analisi delle ricadute scientifiche che hanno fatto seguito alla pubblicazione del Symbolisme. Mi sembra che si possano individuare due parti principali nell'introduzione storiografica. Nella prima, i curatori ripercorrono con un'acribia considerevole la nascita e le tappe principali del progetto cumontiano, i lavori preliminari 
che ne hanno permesso la realizzazione, gli obiettivi del volume, la sua ricezione e la sua fortuna. La lettura di queste pagine permette di immergersi nel dibattito accademico della prima metà del XX secolo, nei rapporti tra Cumont e gli altri studiosi dell'epoca, nelle scoperte archeologiche che contribuirono ad attirare l'attenzione dello studioso belga sull'importanza delle fonti materiali. II volume sul Symbolisme viene così ricollocato nella complessa rete di relazioni accademiche intrecciate da Cumont, tra Roma, Parigi, Bruxelles, Yale, etc., mettendone in evidenza i punti di forza e i limiti. Ampio spazio viene dato all'analisi della ricezione dell'opera: le recensioni, apparse negli anni immediatamente successivi la pubblicazione, mostrano un apprezzamento quasi unanime da parte della critica (con la sola eccezione dei rilievi critici di Henri Marrou e Arthur D. Nock, ampiamente commentati dai curatori del volume). Si ripercorre anche il ruolo giocato dal Symbolisme nelle opere pubblicate dopo la morte di Cumont, rivelando cosi l'interesse per questo lavoro da parte di alcuni studiosi di primissimo ordine nel campo degli studi iconografici del Novecento, come Erwin Panofsky, Martin P. Nilsson e Friedrich Matz. La panoramica proposta dai curatori considera anche l'influenza esercitata dal testo di Cumont in studi più recenti, come quelli di Robert Turcan, Ramsay MacMullen, fino alle riflessioni di John Scheid. Certo, il saggio di Cumont non ha sempre goduto di una buona ricezione in ambito accademico; anzi, negli ultimi decenni il rifiuto nei confronti delle teorie cumontiane è stato pressoché unanime, a causa di una diffidenza diffusa nei confronti di una lettura allegorica e religiosa dei miti rappresentati nei monumenti funerari romani.

Per questo motivo, nella seconda parte dell'introduzione, gli autori propongono una "riabilitazione" del testo di Cumont, vale a dire una riflessione sull'attualità e l'utilità che le teorie di Cumont possono ancora rivestire per i nostri studi contemporanei. Di grande interesse sono in particolare le pagine dedicate al rapporto tra i sarcofagi e la società romana: la ricostruzione del contesto stori- co e sociale proposta dai curatori permette di correggere l'idea cumontiana secondo la quale l'interpretazione filosofica dei miti rappresentava la seule foi des classes élevées (p. 2). È opportuno attribuire una buona parte della produzione dei sarcofagi alla classe degli affrancati la cui importanza era crescente nell'amministrazione imperiale a partire dalla metà del I secolo d.C. e i cui interessi filosofici erano legati alla loro origine, spesso orientale. Commentando le pagine del Symbolisme, i curatori propongono al lettore anche una storia dell'origine e della diffusione dei sarcofagi nel mondo romano, attraverso una rassegna storiografica che si interroga sulla componente sociale delle famiglie che avevano scelto questa forma di sepoltura. Questa sezione assume i tratti di un saggio a sé stante sulla produzione artistica di ambito funerario nell'impero dei primi secoli.

L'introduzione storiografica apporta un contributo fondamentale al dibattito sulla lettura delle immagini e sul loro rapporto con le religioni del mondo antico. Al di là delle interpretazioni delle singole immagini e dei singoli miti, in effetti, la questione di fondo studiata da Cumont è quella del rapporto tra "immagini" e "credenze", tra rappresentazioni iconografiche e pensiero religioso: le raffigurazioni contenute nei sarcofagi e nelle stele funerarie sono da considerare come semplici ornamenti e decorazioni o sono, invece, l'espressione simbolica, nella forma linguaggio visuale, di concezioni filosofiche e teologiche? Sebbene di primo acchito si potrebbe essere tentati di considerare $\mathrm{Cu}$ mont come un sostenitore della tesi "religiosa" (come ad esempio aveva fatto Arthur D. Nock), i curatori del volume mostrano che la questione è più complessa e la posizione di Cumont meno definita e, per questo motivo, ancora utile per la nostra riflessione.

Non si può non salutare con riconoscenza il lavoro di Janine et Jean-Charles Balty la cui ricchezza di informazioni (storiche, archeologiche e bibliografiche) e la cui profondità di analisi fa sì che il saggio di Cumont possa godere di nuova considerazione nell'attuale dibattito scientifico. 